Research Article

\title{
Human Falling Detection Algorithm Based on Multisensor Data Fusion with SVM
}

\author{
Daohua Pan $\mathbb{D}^{1,2}$ Hongwei Liu ${ }^{1}{ }^{1}{ }^{1}$ Dongming Qu, ${ }^{3}$ and Zhan Zhang ${ }^{1}$ \\ ${ }^{1}$ School of Computer Science and Technology, Harbin Institute of Technology, Harbin 150001, Heilongjiang, China \\ ${ }^{2}$ Department of Electronic and Information Engineering, Heilongjiang Vocational College for Nationalities, Harbin 150066, \\ Heilongjiang, China \\ ${ }^{3}$ Department of Financial Technology, China Construction Bank, Harbin 150001, Heilongjiang, China
}

Correspondence should be addressed to Daohua Pan; pandaohua@ftcl.hit.edu.cn and Hongwei Liu; liuhw@hit.edu.cn

Received 23 September 2020; Revised 14 October 2020; Accepted 17 October 2020; Published 31 October 2020

Academic Editor: Jianhui Lv

Copyright (c) 2020 Daohua Pan et al. This is an open access article distributed under the Creative Commons Attribution License, which permits unrestricted use, distribution, and reproduction in any medium, provided the original work is properly cited.

\begin{abstract}
Falling is a common phenomenon in the life of the elderly, and it is also one of the 10 main causes of serious health injuries and death of the elderly. In order to prevent falling of the elderly, a real-time fall prediction system is installed on the wearable intelligent device, which can timely trigger the alarm and reduce the accidental injury caused by falls. At present, most algorithms based on single-sensor data cannot accurately describe the fall state, while the fall detection algorithm based on multisensor data integration can improve the sensitivity and specificity of prediction. In this study, we design a fall detection system based on multisensor data fusion and analyze the four stages of falls using the data of 100 volunteers simulating falls and daily activities. In this paper, data fusion method is used to extract three characteristic parameters representing human body acceleration and posture change, and the effectiveness of the multisensor data fusion algorithm is verified. The sensitivity is $96.67 \%$, and the specificity is $97 \%$. It is found that the recognition rate is the highest when the training set contains the largest number of samples in the training set. Therefore, after training the model based on a large amount of effective data, its recognition ability can be improved, and the prevention of fall possibility will gradually increase. In order to compare the applicability of random forest and support vector machine (SVM) in the development of wearable intelligent devices, two fall posture recognition models were established, respectively, and the training time and recognition time of the models are compared. The results show that SVM is more suitable for the development of wearable intelligent devices.
\end{abstract}

\section{Introduction}

Population aging problem is increasingly prominent, and the technology to help adapt to the aging has been widely concerned. Due to the aging of physiological structure and the decline of physical function for the elderly, the probability and frequency of accidental falls in the elderly are very high. The development of human fall detection technology has practical application value. In daily life, especially in wet places such as the kitchen, bathroom, and toilet, elderly people slip easily. If the elderly suffer from cerebral hemorrhage, heart disease, and other diseases, falls may also threaten their life. Therefore, the detection of falls is particularly important for the elderly. As one of the key components of intelligent devices, sensors have been widely used in the field of smart portability. With the expansion of the application range, more and more high requirements are put forward for the preparation of sensors with high sensitivity, high precision, and flexibility. At present, the fall detection based on wearable sensor is relatively accurate, which usually does not affect the detection results due to environmental changes. It is flexible and easy to use. It can also install sensors such as heart rate and blood pressure to monitor the physical condition of the elderly in real time. The multisensor attitude detection system combines multiple data together to get the prediction results and real-time output. Compared with the detection system based on a single sensor, the cooperative use of multiple sensors can further improve the accuracy and reliability of prediction. 
The research of sensors and fall prevention is deep. Wang's research introduced the design and implementation of RT fall (Real-Time fall). The system was a real-time, noncontact, low-cost, and accurate indoor fall detection system using commercial Wi-Fi equipment. Using the phase and amplitude of fine-grained channel state information (CSI) that can be accessed in commercial Wi-Fi devices, RT fall achieved the goal of real-time automatic segmentation and fault detection for the first time. The sharp drop of power spectrum of falls was found in time-frequency domain, which provided a new idea for new feature extraction and precise fall detection. However, Wang's research was biased towards theory and had no practical support [1]. He established a motion model based on three-dimensional acceleration and gyroscope to analyze the difference between activities of daily living (ADLs) and falls. At the same time, He introduced k-NN algorithm and sliding window technology and developed an intelligent fall detection and alarm system composed of wearable motion sensor board and smart phone, but there was a problem of low sensitivity [2]. Thanh had developed a low-cost fall detection system to accurately detect accidental falls in the elderly. He used fall detection algorithm to compare acceleration with lower and higher fall thresholds to accurately detect falls. However, the system performance therein was not good, and the energy requirements are high, and the practicability was not high [3]. Kepski proposed a fall detection algorithm based on depth map sequence and wireless inertial sensor data worn by the monitored person. Kepski s experimental verification was carried out on a freely obtained dataset consisting of synchronized depth and acceleration data. Kepski had done a lot of experiments in the scene. A static camera faced the scene, and an active camera observed the same scene from above. Experiments on human detection, tracking, and realtime fall detection verified the effectiveness and reliability of the proposed method. However, the cost of this method was high, so it was not suitable for large-scale promotion [4].

In this study, the effectiveness of the fall detection algorithm based on multisensor data fusion is verified by using the data of 100 volunteers simulating falls and daily activities and whether the increase of sample number in machine learning can increase the recognition rate of the system. The comparison of random forest algorithm and support vector machine algorithm, which is more suitable for wearable devices, to predict the trend of falls in recognition rate and recognition time is of great significance for the elderly to drop down.

\section{Wearable Sensor and Fall Detection Algorithm}

2.1. Wearable Sensor. Wearable devices integrate sensors into monitoring devices in real time. When the human body falls down, the alarm can be triggered in real time. The advantages of this method are obvious, not limited by time and space, and convenient transportation and low cost.
2.1.1. Flexible Materials. Common sensors are composed of rigid and noncurved substrates, such as those with silicon substrates. The current sensors have been widely used in our real life, but there are also some shortcomings, such as stiffness, sensitivity, and flexibility. Flexible sensor has flexibility; because of its wear, it needs to use flexible materials, such as some elastic staples, threads, or fiber fabrics; organic polymer also has corresponding applications [5]. The main substrates used are PET and REN because they have the advantages of transparency and low cost compared with other organic polymers. In the development of wearable health medical devices, PDMS, PEN, PI, and P are often used in the development of flexible sensors. The sensor electrode is composed of new driving materials, such as carbon nanomaterials and metal nanoparticles. The starting materials are graphene, carbon nanotube (CNT), carbon fiber [6], silver, gold, and nickel. PDMS, PET, PEN, and PI are commonly used as insulating substrates for the development of flexible sensors. The differences between these polymer materials mainly lie in Young s factor and refractive index. We usually choose the appropriate manufacturing process according to the size of flexible sensors [7].

2.1.2. Types of Sensing Indicators. Wearable sensors are mainly used to detect human normal indicators, so they have been widely used in our lives. Generally speaking, through the detection of human normal indicators, such as blood pressure, cholesterol content, and $\mathrm{pH}$ value, testing them can put forward corresponding reference suggestions for human health. The main application tools in life are electrochemical sensors, pressure and stress sensors, and magnetic field sensors.

2.1.3. System Composition and Characteristics. At present, wearable sensors to detect falls usually use three-axis acceleration sensors [8]. By integrating the acceleration sensor into relevant wearable devices, the human body wears it and collects motion information from the human body. After analyzing and processing the collected information, it can be judged whether the human body falls [9]. For example, using the possibility and working principle of the three-axis acceleration ADXL345, the three coordinates of ADXL345 correspond to the left and right, the front and rear directions of the human body, and the change of acceleration in each direction. When the human body falls, the acceleration in all three directions will change. Acceleration changes in three directions should be calculated and appropriate thresholds should be set to determine whether the elderly fall [10]. The fall detection based on wearable sensor is relatively accurate and generally does not affect the detection results due to environmental changes, and it is flexible and easy to use. Sensors such as heart rate and blood pressure can also be installed to monitor the physical condition of the elderly in real time. For the sensor beam fall detection algorithm, the commonly used algorithm should specifically include the 
methods based on the following contents: time domain analysis and human fall behavior recognition method based on threshold analysis. The human fall process algorithm based on time series analysis can be used for the prediction and detection of human falls not only to predict whether human falls will occur but also to identify human falls [11].

\subsection{Fall Detection Algorithm Based on Multisensor Data Fusion}

\subsubsection{Analysis of Fall Process and Design of Detection} Algorithm. According to the impact strength to the ground, falls can be divided into "hard fall" and "soft fall." "Hard fall" refers to a fall resulting from physical illness due to impact. "Soft fall" refers to a fall that does not cause harm due to low impact strength, because the elderly fall slowly against the wall. Due to the greater threat of hard fall to the body, this paper focuses on the analysis of this kind of fall. According to the principle of mechanics, when the projection of the center of gravity to the ground cannot fall into the supporting surface, the mechanism of body force balance will be broken. If the center of gravity or supporting surface is not adjusted in time, a fall may occur. This imbalance state will be manifested in two forms: the body lands at certain acceleration, and the body posture angle changes [12]. This fall can be divided into four stages:

(1) The fall begins: the fall does not occur, but the balance was destroyed, and the body acceleration and position angle do not change at this time.

(2) Down phase: from the body losing balance to the end of contact with the ground, the acceleration value changes, and the stop angle and angular velocity of the object rotating around the axis change.

(3) Impact phase: from the moment when the body contacts the ground until it stops on the ground, in this stage, the acceleration value in the middle of the body will change dramatically and bear the maximum impact, which is also the stage of causing damage [13].

(4) Immobility phase: the postimpact phase is usually characterized by lying on the ground for a period of time.

The whole process (including four steps) of fall detection algorithm based on multisensor and multiparameter data fusion is summarized as follows: SV and BVA values determine whether the fall has started. When the data of five consecutive points exceed the limit a, it shall be regarded as the beginning of decline. At this time, the stop angle values of Pitch1, Roll1, and Yaw1 are recorded to determine the descent phase. When the data of five consecutive points exceeds the B limit, the descent phase occurs and the timer is activated. BVA and SVD were used to determine the impact stage. When the data of five consecutive points exceeds the limit $\mathrm{C}$, the collision phase occurs. Disable the timer and read the value to check whether it is less than 1 second. Use BVA, Pitch, Roll, and Yaw to determine the immobility phase. When 400 consecutive data points are within a specific range $\mathrm{D}$, they are in the immobility phase, and the position angle values of Pitch2, Roll2, and Yaw2 are recorded [14]. The two recorded stop angle values are used to determine the type of fall.

2.2.2. Analysis of Control System Design Requirements. When it is determined that a human body has fallen, the system must be able to send a text message to a specific person through GPRS to send an alarm. Therefore, the basic program of the controller is as follows: the inertial sensor continuously detects the data and sends it to the MCU for processing. According to the postposition detection algorithm, the MCU uses the processed data to determine the current rear position. The fall recognition based on threshold value is proposed. If it fails, decide whether to perform alarm operation, such as voice unit, engine unit, and GPRS unit $[15,16]$. In addition, if the current device is connected to the mobile phone through Bluetooth, the mobile phone application can send parameter adjustment instructions to the device, such as angle adjustment command, engine vibration command, and size command voice unit $[17,18]$. When using, if the hardware adds other updated functions or there are problems when using the device, you can remotely update the latest program in GPRS through the server. Through the analysis of workflow, the design controller must have the following four functions:

(1) Communication function, advanced computer communication function, and mobile application server.

(2) Storage operation, parameter control command, and bad attitude record storage.

(3) The operation of receiving and processing data, receiving data from inertial sensors, and processing to detect falls.

(4) Input and output functions, including ADC power detection, IO port operation, engine, and voice drive.

2.2.3. Data Fusion Algorithm. The classical data fusion algorithms include multidimensional scaling (MDS) and Isomap, while the traditional data fusion algorithms include principal component analysis (PCA). MDS algorithm displays the points in the high-dimensional coordinate system to the low-dimensional coordinate system and ensures the minimum distance between the two points. After the data is reduced, the best high-dimensional local space is selected for dimensionality reduction. In MDS algorithm, the distance table of sample $m$ in the original space is $D$, and the distance table of lower space is $Z$. After selecting the initial point, the gradient method is used to solve the low-dimensional mapping, so that DZ is the minimum. MDS does not need to make assumptions on data and can process large-scale data, but the calculation cycle is long, and the samples will fall into local optimum. Isomap uses the same basic algorithm as MDS, while Isomap uses geographic distance to reflect the low dimension of the actual multistructure calculation. For the $n$-dimensional matrix, after the space input distance DX 
$(I, J)$ between two points is specified by Isomap, the shortest path DG $(I, J)$ is calculated by Dijkstra algorithm, which is used to estimate the geological distance. Finally, DG uterine distance was used as the input of MDS to obtain the result of reducing the size $[19,20]$. When the number of $N$ vectors increases, the distance between the two points is closer to the geographical distance, but it will last longer; if $n$ is too small, the geological distance will not be accurate.

PCA algorithm is called basic element analysis, which uses linear transformation to transform the original data into a new coordinate system to compress the proportion of the original data [21]. For the initial $M$-dimension and $X$ dimension table, the algorithm must find the interface table and then find the attributes through the combination table. Then, according to the attributes, they are sorted by row from top to bottom, and the first row $K$ is taken to form the matrix $P ; y=\mathrm{PX}$ is the result of dimension reduction of dimension $K$. The algorithm runs fast, but it is difficult to deal with nonlinear data.

2.3. Support Vector Machine and Random Forest. Mechanical learning is a multidisciplinary interdisciplinary subject that integrates probability theory, statistics, approximation theory, and other related theories. Its research scope includes data mining, computer vision, and speech recognition [22]. At present, there are many kinds of mechanical learning algorithms. For wearable multisensor devices, support vector machine or random forest is recommended.

2.3.1. Support Vector Machine. This is a two-layer recognition method. For a dataset, the linear classifier with the largest separation distance in its feature space is found. The purpose is to maximize the distance between the two categories and develop it into a method to solve problems. When the data is linearly inseparable, the current data must be transformed into high-dimensional space for effective classification. In the support vector machine algorithm, the function that can complete the dimension mapping is called the core function, which can solve the most complex problems, operate the internal products in the dimension space, and enable the data to be classified in the dimension space [23]. The basic principle is to transform the two types of data to be classified into an internal carrying volume and a dimension space through core function transformation. At present, the commonly used kernel functions include linear kernel function, polynomial kernel function, radial basis function (RBF) kernel function, and Fourier kernel function [24].

As shown below, formula (1) is a linear kernel function. For linear kernel function, there is no special parameter to be set.

$$
K(x, y)=x \cdot y \text {. }
$$

Formula (2) is a polynomial kernel function. For polynomial kernel function, $D$ is used to set the highest degree of this term of polynomial kernel function. The default value is $3 . C$ is used to set coef0 in kernel function, and the default value is 0 .

$$
K(x, y)=(x \cdot y+c)^{d} \text {. }
$$

Formula (3) is a radial basis function. For radial basis function, gamma is used to set gamma parameter in kernel function. The default value is $1 / K$ ( $K$ is the number of categories).

$$
K(x, y)=\exp \left(-\gamma\|x-y\|^{2}\right)
$$

Formula (4) is a Fourier kernel function. For the Fourier kernel function, $q$ is used to set the gamma parameter in the kernel function. The default value is $1 / K(K$ is the number of categories).

$$
K(x, y)=\frac{1-q^{2}}{2\left(1-2 q \cos (x-y)+q^{2}\right)} .
$$

Among these kernel functions, the operation of kernel basis is more complex than that of polynomial kernel, which may have a better influence on the relationship between classification and classification and can deal with the characteristics of linear indivisible data. Therefore, this paper selects the fundamental kernel function as the core function of support vector machine algorithm.

2.3.2. Random Forest. This is a multidecision tree classifier. Its output category is created after comparing the output results of all decision trees. The advantage of random forest is that it can create a high-precision classifier, process a large number of input variables, evaluate the significance of variables, and balance the error classification of unbalanced material sets. Based on the decision tree theory, $N$ data samples are randomly selected from the original dataset to form a sample subset, which is determined by the sample subset. According to the above steps, create other decision trees to form a random forest. When the forest category is evaluated most, it is determined according to the data of the new classification tree. The random forest algorithm is an improved algorithm based on the decision tree algorithm. It contains multiple decision trees, and the creation of each decision tree depends on the samples collected independently each time. The process of random forest algorithm should be divided into the following steps:

(1) Select random data sample $n$ from the original dataset.

(2) The random $K$ attributes are derived from all attributes, and a decision tree is created based on the samples selected in $(L)$ based on these attributes.

(3) Repeat (1) and (2) for a total of $M$ times to generate a decision tree and create a random forest.

(4) When classifying new data, each tree will make a decision, and all the decision tree votes are used to determine the final classification result.

Compared with other mechanical learning algorithms, random forest has the following advantages: 
(1) It can process dimensional data and identify relationships between them.

(2) The classification model has certain stability for effective evaluation of missing data.

(3) Random forest has a strong generalization effect.

(4) The random sampling makes the classification model avoid overpositioning.

\section{Experimental Design and Parameter Selection}

3.1. Experimental Methods. This paper designs a fall detection system based on multisensor data fusion and analyzes the four stages of fall. By using the method of data fusion, three characteristic parameters representing human acceleration and posture change are extracted, and a fall detection algorithm based on threshold crisis is designed.

To be safe, we recruited 100 college students to simulate the experiment of falling. Their average age was 21 years, and their average height was $172 \mathrm{~cm}$. A total of 100 volunteers were tested on a $10 \mathrm{~cm}$ protective mat for forward, backward, and lateral three times. The multiple sensors were located at the waist. Each volunteer also completed a series of daily actions such as walking (five steps), standing (3s), sitting $(5 \mathrm{~s})$, squatting down (3s), lying (10s), going upstairs (ten steps), and going downstairs (ten steps). The 3D acceleration and angle data were collected from the fall detection system to verify the effectiveness of the algorithm. Manual inspection was required to ensure that the collected data should be available. According to the collected data, the data waveform of each one, energy is calculated. After preprocessing, the corresponding features are derived from each station data, and these features are taken as the original sample set.

3.2. Selection of Characteristic Parameters. According to the analysis of falling process, the following acceleration characteristic parameters were extracted:

$$
\mathrm{SV}=\sqrt{A_{X}^{2}+A_{Y}^{2}+A_{Z}^{2}}
$$

Among them, $A_{X}, A_{Y}$, and $A_{Z}$ are the outputs of accelerometer in three directions. SV reflects the instantaneous acceleration of the body in the process of falling, which is composed of dynamic and static parts. Dynamic acceleration (SVD) is the high-frequency part of SV, which reflects the intensity of acceleration change, and can be used for impact judgment, which is obtained by formula (6); static acceleration refers to the acceleration of body at rest, usually $1 \mathrm{~g}$.

$$
\operatorname{SVD}=\sqrt{\left(A_{X}^{(1)}\right)^{2}+\left(A_{Y}^{(1)}\right)^{2}+\left(A_{Z}^{(1)}\right)^{2}}
$$

Among them, $A_{X}^{(1)}, A_{Y}^{(1)}$, and $A_{Z}^{(1)}$ are the data of $A_{X}, A_{Y}$, and $A_{Z}$ after passing through the third-order high-pass Butterworth filter with cut-off frequency of $0.2 \mathrm{~Hz}$.

The vertical acceleration dynamic quantity (BVA) of the body is obtained by

$$
\mathrm{BVA}=\frac{\mathrm{SV}^{2}-\mathrm{SVD}^{2}-G^{2}}{2 G} .
$$

The peak value of BVA appears during impact, which can be used to explain the oscillation behavior during impact. BVA can be used to determine the impact.

3.3. Validation of Algorithm Effectiveness. In order to verify the effectiveness of the proposed algorithm, experiments are carried out. Firstly, the relevant threshold is designed; then the data of volunteers' actions are collected and processed by the above algorithm, the performance of characteristic parameters is analyzed, and the accuracy rate SE and recognition rate SP are calculated. SE is defined in formula (8) and SP is defined in formula (9).

$$
\mathrm{SE}=\frac{D}{S} \times 100 \%
$$

where $D$ is the number of successful detections and $S$ is the total number of falls.

$$
\mathrm{SP}=\left(1-\frac{A}{B}\right) \times 100 \%
$$

Among them, $A$ is the number of times that was mistakenly detected as falling and $B$ is the total number of daily actions.

Sensitivity is the capacity to detect a fall. It is given by the ratio between the number of detected falls and the total falls that occurred:

$$
\text { Sensitivity }=\frac{\mathrm{TP}}{\mathrm{TP}+\mathrm{FN}} \text {. }
$$

Specificity is the capacity to avoid false positives. Intuitively it is the capacity to detect a fall only if it really occurs:

$$
\text { Specificity }=\frac{\mathrm{TN}}{\mathrm{TN}+\mathrm{FP}}
$$

where the meanings of TP, FN, FP, and TN are shown in Table 1.

\section{Analysis of Experimental Results}

4.1. Performance of Multifeature Parameter Fall Detection Algorithm in Falls. The performance of the algorithm is shown in Table 2 and Figure 1. If a feature is detected, it will be accumulated once.

It can be seen from Table 2 and Figure 1 that the algorithm proposed in this paper has successfully detected 175 times (180 times in total) and falls ( $\mathrm{SE}=96.69 \%)$. It has good performance in front and back falls, but only $90 \%$ of them fall in the horizontal direction, which is mainly due to the high threshold value and the missing detection in the fall. The main reason is that the fall effect is relatively "slow" due to the support force when the foot falls to the side. Both SVD and BVA can track the fall stage. Because the stop angle changes before and after the fall, it recovers. Combined with these four stages, the accuracy of the algorithm is as high as 
Table 1: Possible outputs of a fall detection system.

\begin{tabular}{lcc}
\hline & A fall occurs & A fall does not occur \\
\hline A fall is detected & True positive (TP) & False positive (FP) \\
A fall is not detected & False negative (FN) & True negative (TN) \\
\hline
\end{tabular}

TABle 2: Performance of this algorithm in fall.

\begin{tabular}{|c|c|c|c|c|c|}
\hline \multirow{2}{*}{\multicolumn{2}{|c|}{ Action }} & \multicolumn{4}{|c|}{ Characteristic parameters } \\
\hline & & SV & SVD & BVA & Number of successes \\
\hline \multirow{4}{*}{ Fall forward } & The fall begins & 54 & & 60 & 60 \\
\hline & Down phase & & & & 60 \\
\hline & Impact phase & & 60 & 60 & 60 \\
\hline & Immobility phase & & & 58 & 60 \\
\hline \multirow{4}{*}{ Fall back } & The fall begins & 59 & & 60 & 60 \\
\hline & Down phase & & & & 60 \\
\hline & Impact phase & & 60 & 60 & 60 \\
\hline & Immobility phase & & 58 & & 60 \\
\hline \multirow{4}{*}{ Sideways } & The fall begins & 50 & & 60 & 60 \\
\hline & Down phase & & & & 60 \\
\hline & Impact phase & & 60 & 60 & 60 \\
\hline & Immobility phase & & & 60 & 60 \\
\hline
\end{tabular}

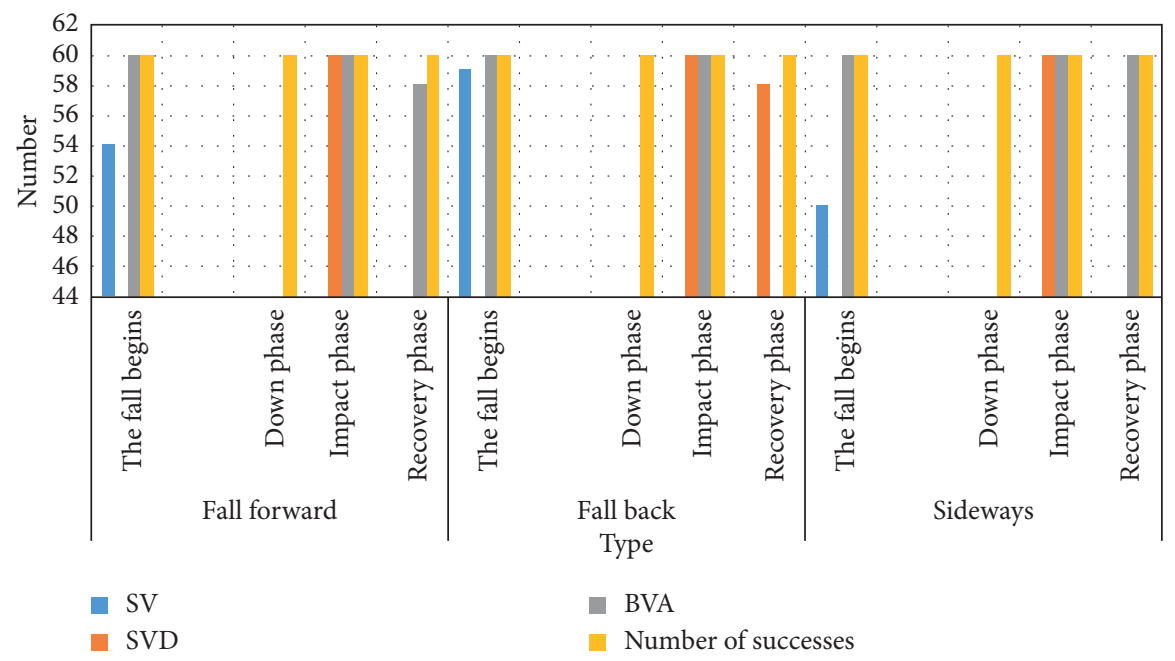

FIgURe 1: Performance of this algorithm in fall.

100\%. The multifunctional parameters under multiple sensors can significantly improve the measurement accuracy.

\subsection{Probability of Each Parameter Exceeding the Limit in Daily} Activities. In the daily activities of the algorithm, the probability of each parameter exceeding the limit value is shown in Table 3 and Figure 2.

From Table 3 and Figure 2, we can see that the probability of each parameter in the algorithm exceeds the limit in daily activities; then a group of daily activities is equivalent to 10 falls in this study, and the false detection rate $S_{i j}$ of each characteristic parameter is calculated, as shown in formula (10).

$$
S_{i j}=\frac{N_{i j}}{10 \times 10} \times 100 \%
$$

Among them, $i=$ fall start, fall, impact, and recovery; $j=\mathrm{SV}, \mathrm{BVA}$, and SVD; $N_{i j}$ is the number of false detections. As a result of 6 times of false detection, $S_{i j}$ is $6 \%$ and SP value is $94 \%$. It can be seen from Table 2 that the false alarm rate of acceleration related characteristic parameters is higher than that of angle. SV has the highest false alarm rate, while BVA has low false alarm rate. This is because BVA is the dynamic quantity of SV, and the daily activity changes slowly. Therefore, it is appropriate to increase the weight of BVA parameters in daily behavior assessment. Combined with Sections 4.1 and 4.2 , we have falls and daily activities data of 
TABLe 3: Performance of this algorithm in daily behavior.

\begin{tabular}{lccccc}
\hline & \multirow{2}{*}{ Action } & & \multicolumn{3}{c}{ Characteristic parameters } \\
& & SV (\%) & SVD (\%) & BVA (\%) & Number of successes (\%) \\
\cline { 3 - 6 } Daily behavior & The fall begins & 13 & 3 & 2 & 13 \\
& Down phase & & 1 & 1 & 2 \\
& Impact phase & & 8 & 5 \\
\hline
\end{tabular}

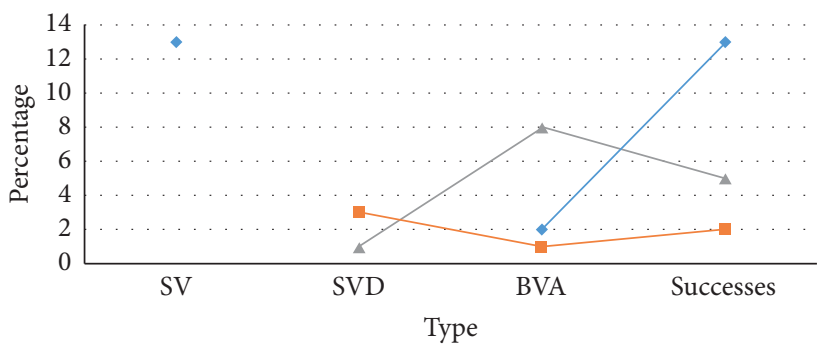

$\rightarrow$ The fall begins

- Impact phase

FIgURe 2: Performance of this algorithm in daily behavior.

100 volunteers to verify the algorithm, with a sensitivity of $96.67 \%$ and a specificity of $97 \%$.

\subsection{Multisensor Fall Detection Based on Support Vector} Machine. Through the 4.1 data acquisition experiment, four kinds of human posture data are obtained, and five kinds of posture features are extracted. These features reflect different points from different angles. According to the human classification of different postures, a fall recognition model based on support vector machine (SVM) algorithm is proposed by controlling the corresponding optimal parameters. The specific steps are as follows:

(1) Human body attitude sensors (including accelerometers and gyroscopes) are used for data acquisition, so as to collect multiple human body data at the same time, including human body triaxial acceleration and angular velocity.

(2) Preprocess the collected original data, using the average filtering method to filter the human posture data, in order to obtain better and clearer data.

(3) The features are derived from all the processed kinds of human body position data (sitting, lying, falling, sliding, squatting down, walking, standing, and going upstairs and downstairs). The features of different positions are taken, including five characteristics of combined acceleration and vertical component of combined acceleration.

(4) In order to build the model based on the derived human pose set, ten times grid crossover and optimization algorithm should be used to determine the best training parameters. To evaluate the model, introduce the test set data to train the autumn attitude recognition model, and calculate the accuracy of the model in the test set.
According to the above steps, 86 groups of features are divided into training set and test set. After determining the optimal parameters, each dataset is randomly mixed and verified five times to ensure that the model has good generalization ability. The experiment was divided into three groups, and the total number of experiments in each group was different, but the number of falls was the same. The numbers of training sets and test sets and the identification results are shown in Figure 3.

As can be seen from the results in Figure 3, when the training set contains different data samples, the recognition results are slightly different. When the training set contains 65 samples and 8 groups of falls, the recognition rate should be $82 \%$. In the training process, the set contains 70 samples, of which 8 groups are falls, and the recognition rate is $86.23 \%$. When the training set contains 75 samples, of which 8 groups are falls, the recognition rate should be as high as $92.15 \%$, and the recognition rate will improve with the increase of the number of samples in the selected education set, which indicates that the recognition ability of the trained model will gradually improve based on a large amount of effective data, thus increasing the possibility of prediction and prevention of falls.

4.4. Comparative Analysis and Selection of Models. In order to compare the applicability of support vector machine and random forest algorithm, two fall detection models were created and compared. 60 datasets are selected as the training set, including 50 normal positions and 10 falling positions. On the basis of the model, the training time of the model and the recognition time of the fall posture are calculated. The results are shown in Figure 4.

As can be seen from Figure 4, the training time of the model based on random forest is 5.5 seconds, the recognition time is 1.45 seconds, and the recognition rate is 


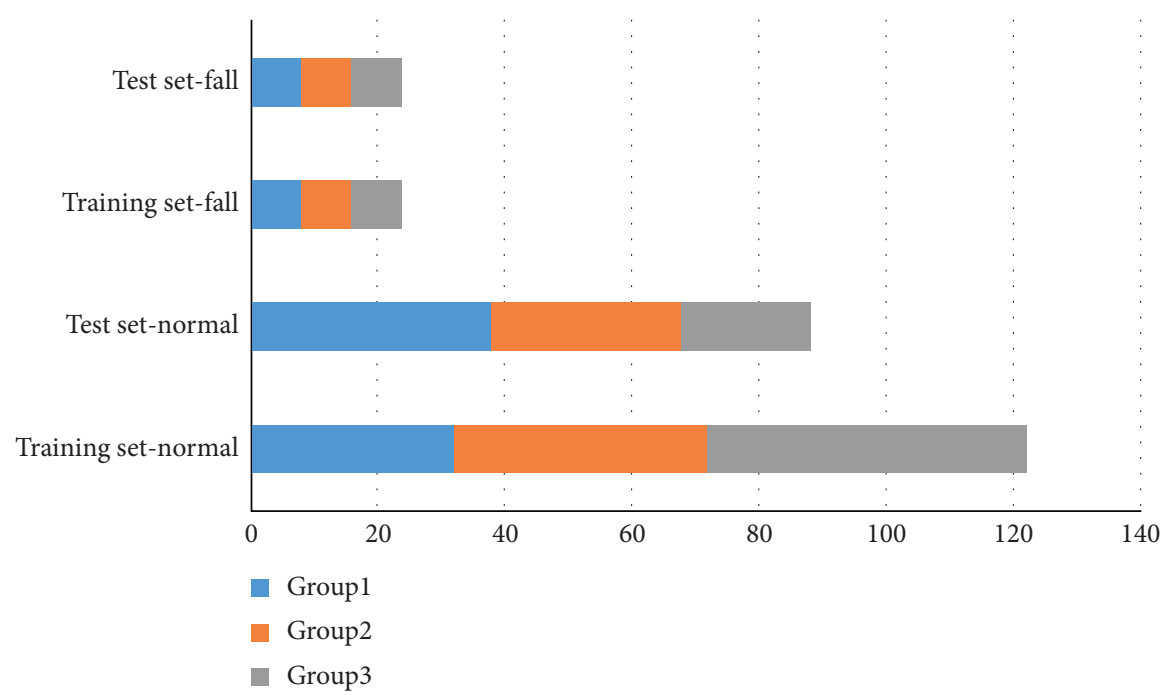

Figure 3: Comparison of fall posture recognition results.

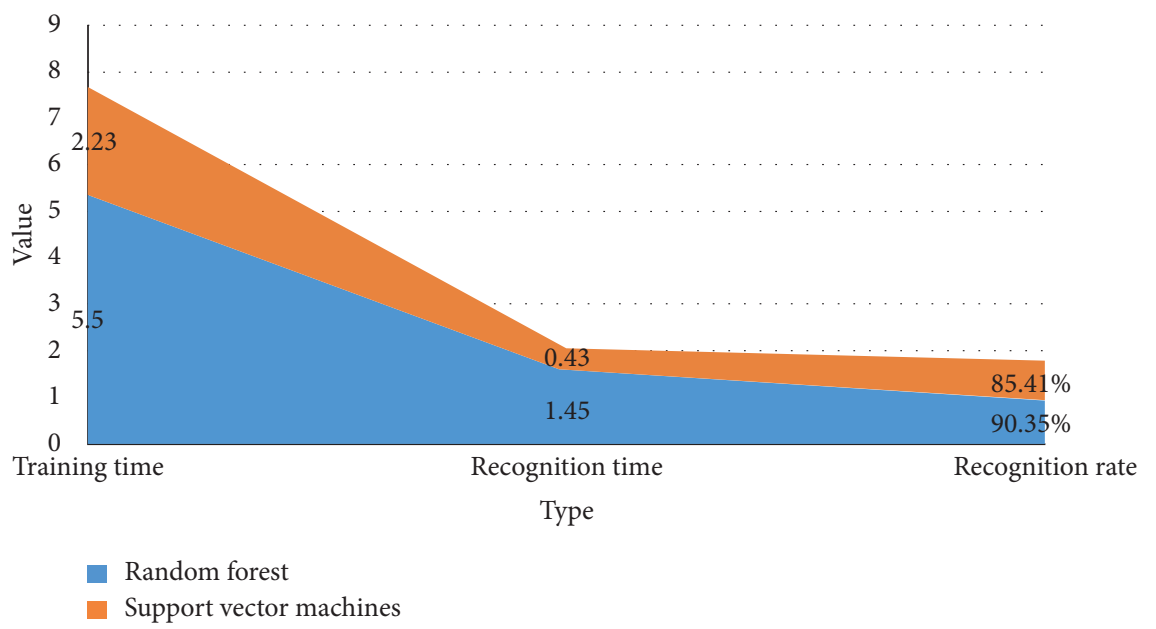

FIgURE 4: Comparison of the recognition results of the two algorithms.

90.35\%; the training time of fall posture recognition model based on support vector machine is 2.23 seconds, the recognition time is 0.43 seconds, and the recognition rate is $85.41 \%$. It can be seen that this machine can make more time-based response to fall recognition than the time-vectorbased recognition algorithm.

\section{Conclusion}

Fall is a common phenomenon in the life of the elderly, and it is also one of the main causes of disease and death of the elderly. The real-time fall detection system can trigger the alarm in time, reduce the waiting time for treatment, and reduce the accidental injury caused by falls. However, in most collision detection systems, only accelerometers are used to design the detection system. However, the algorithm based on single data cannot fully describe the information about body posture changes when falling down. The ultimate goal of this study is to predict the human bodys downward trend sensor based on multidata synthesis fall detection algorithm, so as to effectively detect the occurrence of falls and avoid falls.

In this paper, a data acquisition experiment is designed to preprocess and extract features of human posture data. Then, on the basis of support vector machine algorithm and random forest algorithm, the human posture is recognized, and finally the effective recognition of human posture is realized. In this study, 100 volunteers are collected to simulate falls and daily activities to verify the effectiveness of the algorithm. The sensitivity was $96.67 \%$, and the specificity was $97 \%$, indicating good performance. In the experiment, when the training set contains the largest number of samples and the numbers of fall processing experiments are equal, the recognition rate should reach the maximum value of $92.15 \%$. The accuracy of the model is based on a large amount of valid data. After training, the recognition ability of the system will gradually improve, so as to improve the ability to predict falls. Support vector machine has less training time and recognition time, which brings more warning time and is conducive to prevention. Therefore, 
SVM is more suitable for the system development based on multisensor data fusion.

A fall detection algorithm based on multisensor data synthesis is studied in this paper to improve the accuracy of fall prediction. Wearable devices are very suitable for the elderly and have a very broad application prospect. This study is of great significance to its development.

\section{Data Availability}

All data used to support the findings of the study are included within the article.

\section{Conflicts of Interest}

None of the authors have any conflicts of interest.

\section{Acknowledgments}

This work was supported by the National High Technology Research and Development Program of China (863 Program) under Grant 2013AA01A215.

\section{References}

[1] H. Wang, D. Zhang, Y. Wang, J. Ma, Y. Wang, and S. Li, "RTfall: a real-time and contactless fall detection system with commodity WiFi devices," IEEE Transactions on Mobile Computing, vol. 16, no. 2, pp. 511-526, 2017.

[2] J. He, C. Hu, and X. Wang, "A smart device enabled system for autonomous fall detection and alert," International Journal of Distributed Sensor Networks, vol. 12, no. 2, Article ID 2308183, 2016.

[3] P. V. Thanh, D. T. Tran, D. C. Nguyen et al., "Development of a real-time, simple and high-accuracy fall detection system for elderly using 3-DOF accelerometers," Arabian Journal for Science and Engineering, vol. 44, no. 4, pp. 3329-3342, 2019.

[4] M. Kepski and B. Kwolek, "Event-driven system for fall detection using body-worn accelerometer and depth sensor," IET Computer Vision, vol. 12, no. 1, pp. 48-58, 2018.

[5] S. Amir, Z. Liang, Z. Wang et al., "Novel flexible wearable sensor materials and signal processing for vital sign and human activity monitoring," Sensors, vol. 17 , no. 7, p. 1622 , 2017.

[6] H. Chen, W. Chen, S. Bao et al., "Design of an integrated wearable multi-sensor platform based on flexible materials for neonatal monitoring," IEEE Access, vol. 8, pp. 23732-23747, 2020.

[7] A. Shishido and N. Akamatsu, "Mechanical properties of flexible materials," Kobunshi, vol. 66, no. 7, pp. 362-365, 2017.

[8] G. Li, T. Liu, J. Yi, H. Wang, J. Li, and Y. Inoue, "The lower limbs kinematics analysis by wearable sensor shoes," IEEE Sensors Journal, vol. 16, no. 8, pp. 2627-2638, 2016.

[9] F. Lin, A. Wang, Z. Yan, R. T. Machiko, and X. Wenyao, "A wearable sensor device for unobtrusive gait monitoring in daily life," IEEE Transactions on Industrial Informatics, vol. 12, no. 6, pp. 2281-2291, 2017.

[10] C. Raphael, P. Philipp, and B. Anne-Laure, "Random forest versus logistic regression: a large-scale benchmark experiment," Bmc Bioinformatics, vol. 19, no. 1, p. 270, 2018.

[11] H. F. Nweke, Y. W. Teh, M. A. Al-Garadi, and U. R. Alo, "Deep learning algorithms for human activity recognition using mobile and wearable sensor networks: state of the art and research challenges," Expert Systems with Applications, vol. 105, pp. 233-261, 2018.

[12] X. Huile, L. Jinyi, H. Haibo et al., "Wearable sensor-based human activity recognition method with multi-features extracted from hilbert-huang transform," Sensors, vol. 16, no. 12, p. 2048, 2016.

[13] S. R. Steinhubl, D. Feye, A. C. Levine et al., "Validation of a portable, deployable system for continuous vital sign monitoring using a multiparametric wearable sensor and personalised analytics in an Ebola treatment centre," Bmj Global Health, vol. 1, no. 1, Article ID e000070, 2016.

[14] I. Carpinella, D. Cattaneo, G. Bonora et al., "Wearable sensorbased biofeedback training for balance and gait in Parkinson's disease: a pilot randomized controlled trial," Archives of Physical Medicine \& Rehabilitation, vol. 98, no. 4, pp. 622630, 2016.

[15] Y. Lei, X. Daxi, G. Liquan et al., “A compressed sensing-based wearable sensor network for quantitative assessment of stroke patients," Sensors, vol. 16, no. 2, p. 202, 2016.

[16] W. Liangtian, H. Guangjie, W. Hao et al., "Wearable sensor localization considering mixed distributed sources in health monitoring systems," Sensors, vol. 16, no. 3, p. 368, 2016.

[17] O. Martin, D. Joe, W. Tomas et al., "Mobile app to streamline the development of wearable sensor-based exercise biofeedback systems: system development and evaluation," Jmir Rehabilitation \& Assistive Technologies, vol. 4, no. 2, p. e9, 2017.

[18] H. Lee, H. Chung, H. Ko et al., "Dedicated cardiac rehabilitation wearable sensor and its clinical potential," PLoS One, vol. 12, no. 10, Article ID e0187108, 2017.

[19] A. Buczak and E. Guven, "A survey of data mining and machine learning methods for cyber security intrusion detection," IEEE Communications Surveys \& Tutorials, vol. 18, no. 2, pp. 1153-1176, 2017.

[20] Z. Obermeyer and E. J. Emanuel, "Predicting the future-big data, machine learning, and clinical medicine," New England Journal of Medicine, vol. 375, no. 13, pp. 1216-1219, 2016.

[21] N. D. Sidiropoulos, L. De Lathauwer, X. Fu, K. Huang, E. E. Papalexakis, and C. Faloutsos, "Tensor decomposition for signal processing and machine learning," IEEE Transactions on Signal Processing, vol. 65, no. 13, pp. 3551-3582, 2017.

[22] S. Mirjalili, "Dragonfly algorithm: a new meta-heuristic optimization technique for solving single-objective, discrete, and multi-objective problems," Neural Computing and Applications, vol. 27, no. 4, pp. 1053-1073, 2016.

[23] Z.-M. Yang, H.-J. Wu, C.-N. Li, and Y.-H. Shao, "Least squares recursive projection twin support vector machine for multi-class classification," International Journal of Machine Learning and Cybernetics, vol. 7, no. 3, pp. 411-426, 2016.

[24] Y. Yuan, "Canonical duality solution for alternating support vector machine," Journal of Industrial \& Management Optimization, vol. 8, no. 3, pp. 611-621, 2017. 